\title{
Assesment of Damage of Solar Heterojunction a-SiC/c-Si Induced by Neutron Radiation
}

\author{
Milan Perný ${ }^{1)}$, Vladimír Šály ${ }^{1)}$, Miroslav Mikolášek ${ }^{1)}$, František Janíček ${ }^{1)}$, Vladimír Kujan ${ }^{1)}$, \\ Juraj Packa ${ }^{1)}$ and Jozef Huran ${ }^{2)}$ \\ 1) Slovak University of Technology, Faculty of Electrical Engineering and Information Technology \\ Ilkovičova 3, SK-81219 Bratislava, Slovak Republic, e-mail: milan.perny@stuba.sk \\ ${ }^{2)}$ Slovak Academy of Sciences, Institute of Electrical Engineering, Dúbravská cesta 9, 84104 Bratislava, Slovakia, \\ e-mail:Jozef.Huran@savba.sk
}

\begin{abstract}
Amorphous silicon carbide is known as a material resistant except other influences also against radiation. This study investigates the effects of the neutron radiation on electrical characteristics ( $I-V$ characteristic, impedance spectra and obtained dynamic parameters of AC equivalent circuit) of solar cell a-SiC/c-Si heterojunction structure. The heterojunction structure was irradiated using neutrons with neutron fluency $10^{13} \mathrm{~cm}^{-2}$. Existence of neutron induced structural defects, which could be introduced within the semiconductor structure of heterojunction, has been illustrated using DC and AC analyses. The structural defects induced by neutron particle radiation affect mainly the trapping mechanism. The process results in the changes of electronic elements are included into the proposed equivalent circuit.
\end{abstract}

Keywords - amorphous silicon carbide, structural defects, equivalent circuit, dynamic parameters

\section{INTRODUCTION}

The silicon heterojunction (SHJ) solar cell technology is among the promising area of research and development belonging to the second photovoltaic generation. Benefits of this technology are environmentally friendlier solutions, low temperature deposition processes, the possibility of deposition of larger areas and acceptable conversion efficiency [1]. The studied solar structure is in our case consisting of crystalline silicon wafer as a substrate and deposited doped amorphous silicon carbide thin film, together representing SHJ cell. Due to better management of the light absorption [2] (regarded e.g. tandem approaches), higher open circuit voltage [3]compared to the conventional crystalline silicon technology and high conversion efficiency, is this structure considered to be very promising. Recently the record efficiency of $25.6 \%$ [4] was achieved on the symmetrical SHJ HIT cell, which makes this technology the most efficient among the technologies applied on silicon based solar cells.

One of the potential areas of application of these solar structures is the space application. For this reason, it is necessary to carry out an assessment of threats to the functionality of the device resulting from nonstandard operating conditions (temperature, radiation, etc.). Experience shows that not all available solar PV structures are suitable for use in this type of environment. In addition to the standard tests for assessing the effect of temperature on functionality, reliability and efficiency of solar technology it is essential to carry out an assessment for resistance to various types of radiation [5]. In the space environment, solar cells are exposed to different types of radiation, such as protons, alpha particles, heavy ions, neutrons, gamma rays, electrons, and positrons [6, 7].

Neutrons can be found in nature in small amounts almost everywhere. The main sources in the environment are the cosmic rays. Other sources of neutrons are nuclear reactions and spontaneous fission of heavy nuclei. In structural terms, it is a unique particle that has zero charge but also a relatively large mass. Due to neutrality (no electric charge) there is no possibility of a direct ionization of materials. The basic interaction mechanism with the material is elastic collision with atomic nuclei. This result in gradually losses of its energy but also bombed environment can undergo different states of transformation.

Neutrons and other high energy particles which are part of cosmic rays have the ability by means of collisions with atoms in the semiconductor lattice to form structural defects. These defects usually have sufficient energy to create secondary defects. The interaction of secondary defects with dopants or impurities represents recombination centres and the process finally leads to reducing of the minority carrier lifetime [8]. Axness et al. [9] show that the lattice damage and carrier lifetime degradation are spatially dependent.

In this work we describe measurements undertaken to try to identify the behaviour of heterojunction solar cells, in situation when these have been damaged by the neutron radiation. The DC analysis and complex impedance measurement [10] and analysis of measured data known as the impedance spectroscopy (IS) are often used as suitable and useful techniques to assess the extent of damage resulting from the neutron bombardment. The analysis of the measured and fitted data based on the proposed equivalent circuit allows studying of electrical properties and electronic transport within the investigated structure.

The aim of this paper is to provide results of the processes that occur in solar cells after the neutron irradiation and to give a critical approach of the effectiveness of these devices in the environment with neutron radiation. 


\section{EXPERIMENTAL}

To study the influence of irradiation on photovoltaic device structures based on the crystalline silicon and amorphous silicon carbide heterojunction neutron flux irradiation has been applied in our experiment. The powerful neutron source IBR-2 pulsed reactor was used. The incident energy of neutrons was appr. $1 \mathrm{MeV}$. The dose of neutrons was varied from $\sim 10^{11}$ to $\sim 10^{15} \mathrm{~cm}^{-2}$. The results obtained at the chosen sample irradiated with the neutron dose $10^{13} \mathrm{~cm}^{-2}$ are presented here.

A thin a-SiC:H layer was prepared by a plasma CVD deposition. A crystalline silicon p-type wafer of (100) orientation was used as the substrate for the growth. The deposition of a-SiC (thick about $30 \mathrm{~nm}$ ) was from the gas mixture of $\mathrm{SiH}_{4}=10 \mathrm{sccm}, \mathrm{CH}_{4}=3 \mathrm{sccm}, \mathrm{H}_{2}=100 \mathrm{sccm}$, $\mathrm{PH}_{3}=15 \mathrm{sccm}$, and $\mathrm{Ar}=10 \mathrm{sccm}$. The substrate temperature was $230{ }^{\circ} \mathrm{C}$, pressure $100 \mathrm{~Pa}$ and high frequency power of $50 \mathrm{~W}$. The resulting planar conductivity of the $\mathrm{SiC}$ layer was $\sim 10^{-5} \mathrm{Scm}^{-1}$. More details one can find in [11]. The grid finger electrodes of $\mathrm{Al}$ (200 nm thick) as the ohmic contacts were formed using lift off technique on the top side with the amorphous n-type silicon carbide film on the solar cell samples. The other - bottom side of samples - was fully covered by an Al ohmic contact $(\sim 250 \mathrm{~nm}$ thick). The area of the prepared solar cell structures was $1 \times 1 \mathrm{~cm}$. The electrical behaviour of samples and the irradiation influence were studied using an impedance spectroscopy and DC measurements. $I-V$ characteristics were measured in the dark using KEITHLEY 2440. The impedance measurements were performed by the Solartron Analytical Modul within a wide range of AC frequencies from $10 \mathrm{~Hz}$ to $1 \mathrm{MHz}$. Due to quite low planar conductivity which, of course, results in the not sufficient lateral conductivity, it is more suitable to consider an analyzed structure as an a$\mathrm{Si}-\mathrm{C} / \mathrm{c}-\mathrm{Si}$ heterostructure and not as a complete solar cell. The analyses of such structure have still ability to provide important knowledge regarding the radiation hardness of silicon heterojunction a-Si-C/c-Si, which represents the key part of the silicon heterojunction solar cell. So, the measured effects are related only to the area below the metallization; because of this we consider the structure as an $\mathrm{a}-\mathrm{Si}-\mathrm{C} / \mathrm{c}-\mathrm{Si}$ heterostructure.

\section{RESULT AND DISCUSSION}

The measurements and analyses of the current-voltage $(I-V)$ characteristics in the dark were performed. $I-V$ characteristic, measured in the dark, presented in Fig. 1 confirmed barrier behaviour of the prepared samples. Deterioration of the diode properties as a result of the irradiation by neutrons is clearly observable in the forward part of the $I-V$ characteristic presented in Fig. 1.Existence of two linear regions (region 1 for voltage $V<0.4 \mathrm{~V}$ and region 2 for $V>0.4 \mathrm{~V}$ ) from the DC dark measurement, were recognized in a semi-logarithmic scale of the $I-V$ plot. Two diode models were investigated, when the space charge region and the quasi neutral region were taken into account. The space charge region is mostly affected by the interface properties, while the quasineutral region is influenced by the bulk $[12,13]$. Deviation of the $I-V$ curves from the linear shape for higher voltages is caused by the series resistance $R_{1}$.

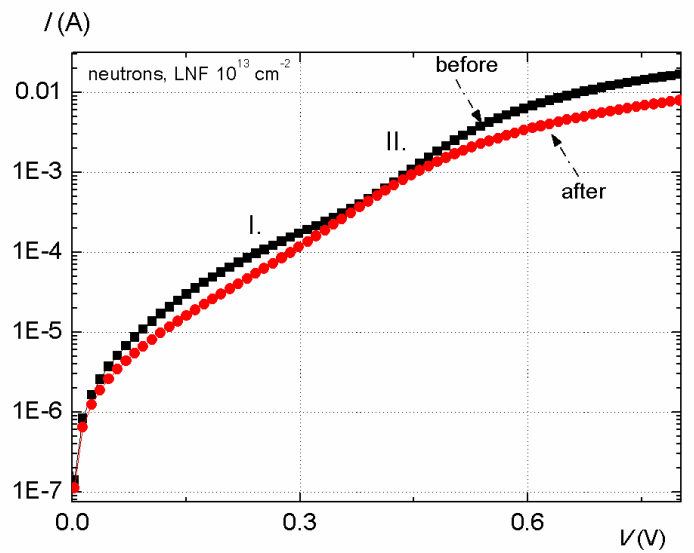

Fig. 1. $I-V$ characteristic in the dark - influence of neutron irradiation.

The ideality factor and series resistance of the $\mathrm{HJ}$ structure was derived using well known procedures from the slope of the dark $I-V$. The results are summarized in Tab. I.

TABLE I.

CALCUlATED a-SiC:H/c-Si DIODE IDEALITY PARAMETER AND SERIES RESISTANCE FOR VOLTAGE REGIONS I. AND II. AT THE DARK

\begin{tabular}{|c|c|c|c|}
\hline & region I. & region II. & $R_{1}(\Omega)$ \\
\hline & $m(-)$ & $m(-)$ & \\
\hline before irradiation & 3.8 & 2.7 & 6.4 \\
\hline after irradiation & 3.14 & 2.9 & 25.08 \\
\hline
\end{tabular}

Unusually large values of the parameter $m(m>2.7)$ as one can see in Tab.I, were determined. Usually the ideality factor $m$ is expected to fall into the range $1<m<2$ [14]. Similarly, like it was pointed out in [15], the high values of $m$ can be caused by defects existing in the quasineutral region, as well as in the junction, and they are responsible for the recombination of the carriers in the junction. Exposing the HJ sample to a neutron bombardment had in our case the effect on increasing the value of the parameter $m$ in the area II from 2.7 to 2.9. It is a consequence of the increase in the number of structural defects due to the neutron bombardment. Increase of the series resistance from $6.38 \Omega$ to $25.08 \Omega$ due to the neutron irradiation was also detected.

The complex impedance was measured using a small $25 \mathrm{mV}$ AC signal before and after the neutron irradiation. The obtained results are presented using frequency dependences of the real and imaginary parts of the measured impedance and they are shown in Fig.2. The influence of the neutron irradiation is easy to quantify. The irradiation of the samples by neutrons affecting the size of the leakage resistance (shift of the saturated $Z$ in the low frequency region) and also distribution of the relaxation times (shift of the minimum $Z^{\prime \prime}$ towards to higher frequencies for higher DC biases). 


$$
Z(\Omega)
$$
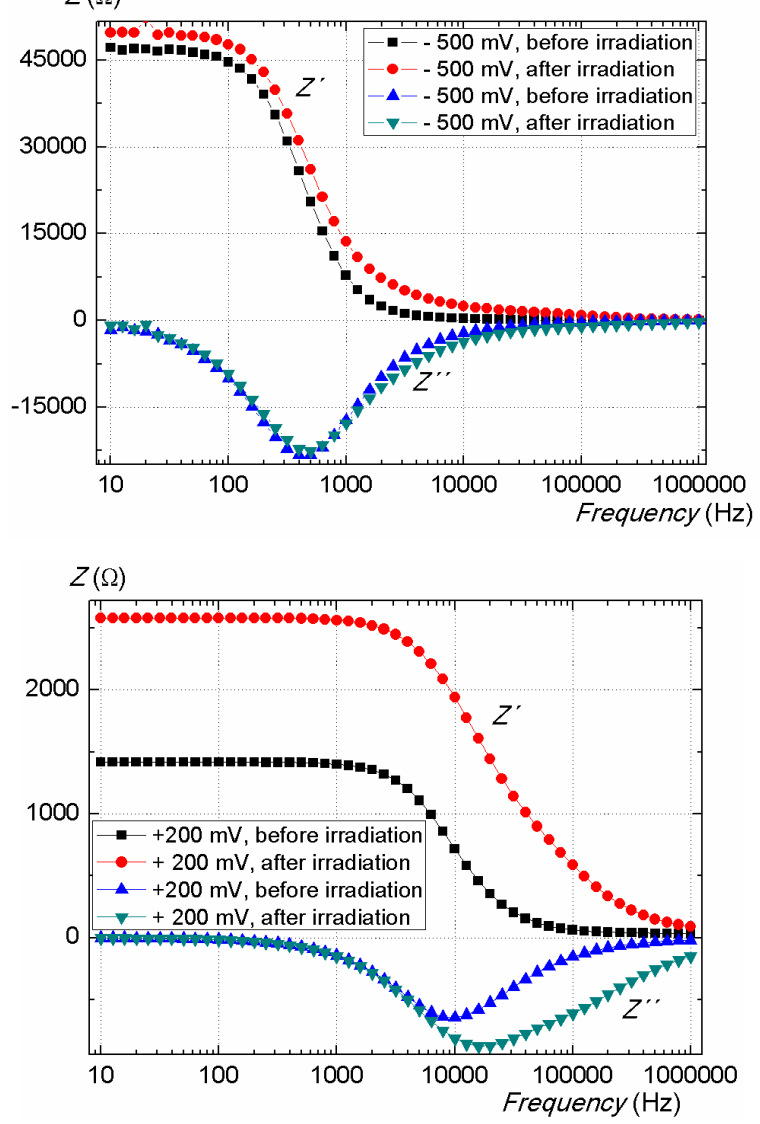
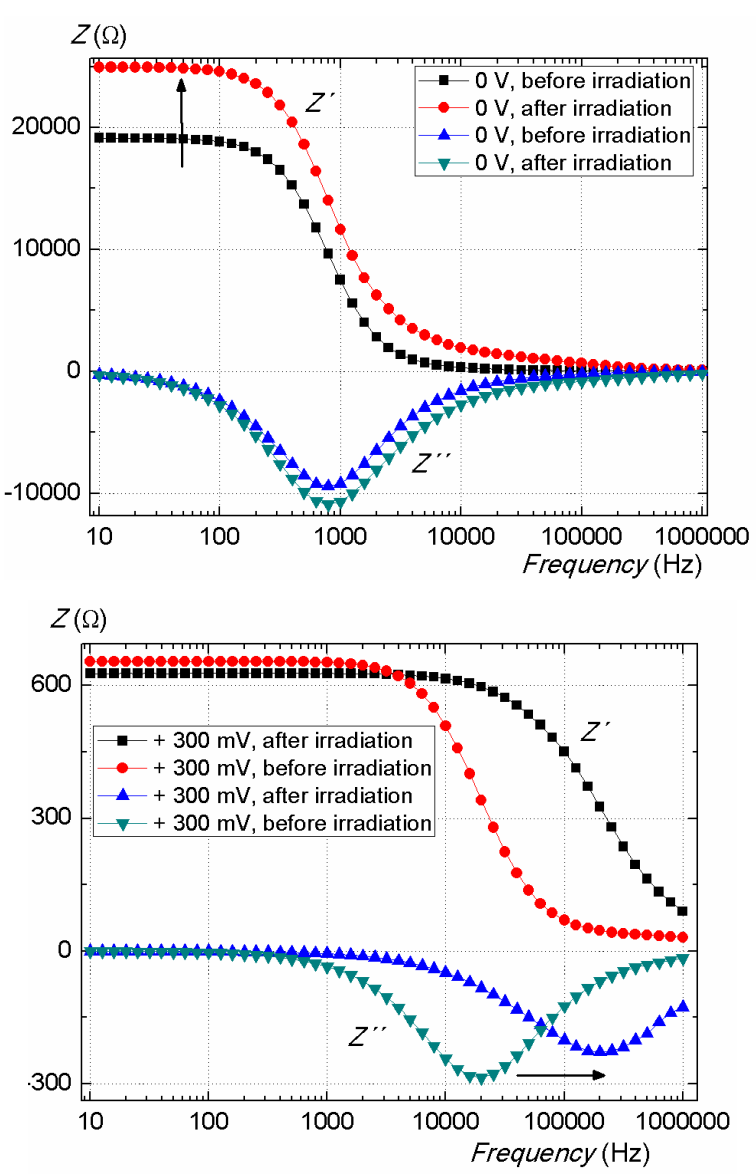

Fig. 2. Real $Z$ and imaginary $Z^{\prime \prime}$ parts of the measured impedance as a function of frequency at a constant DC bias at the dark.

Using numerical simulations before and after the neutron irradiation, the impedance plots were well fitted using the equivalent $\mathrm{AC}$ circuit, as shown in Fig. 3.The impedance component $P$ is a constant phase element (CPE), indicating a distribution of relaxation times.

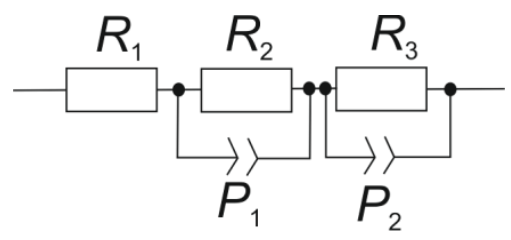

Fig. 3. Proposed AC equivalent circuit.

The constant phase element (CPE in figure denoted as $P$ ) represents a capacitive process with certain frequency dispersion. The electrical impedance of the CPE is expressed as:

$$
Z_{C P E}=1 / P(i \omega)^{n(1,2)}
$$

The subscripts 1 and 2 indicate the high and low frequency regions, usually corresponding to the bulk and surface regions, respectively. The exponents $n_{1}$ and $n_{2}$, each ranging between 0 and 1 , reflect a deviation from the ideal Debye dispersion $(n=1)$. A detailed analysis of the $\mathrm{CPE}$ capacity exponent $\mathrm{n}$ and parallel $\mathrm{AC}$ equivalent circuit resistance on the applied DC bias enables the identification of transport processes in the structure [16,
17]. As can be seen in Fig. 4 the irradiation by neutrons resulted in noticeable drop of the exponent $n_{2}$.

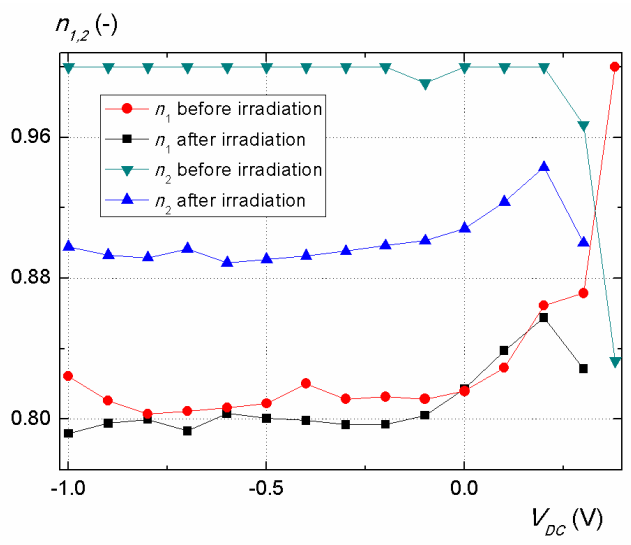

Fig. 4. CPE impedance exponent $n$ as a function of the applied bias.

Taking into account that the exponent $n_{2}$ of the constant phase element represents structural defects in the semiconductor, the decrease of this value is related to the increase in the number of neutrons induced disorders in the deposited thin amorphous layer. The exponent $n_{1}$, which is related to the interface and space charge region, varies due to the neutron exposure minimally.

For a more comprehensive view the impedance response of the constant phase elements at applied bias between $-1 \mathrm{~V}$ and $0.5 \mathrm{~V}$ at the dark is shown in Fig.5. In 
case of the CPE capacitance a slight increase of the capacity $P_{2}$, which is supposed to be associated with the creation of new recombination centres, was observed as a consequence of the neutron bombardment.

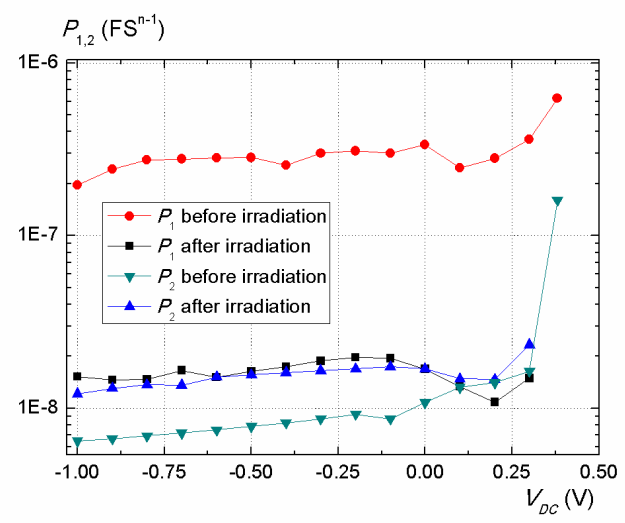

Fig. 5. Impedance results for $\mathrm{CPE}$ capacitances $P_{1}, P_{2}$ as a function of the applied bias before and after neutron irradiation.

Fig.6shows the dependence of the obtained parallel resistances $R_{2}$ and $R_{3}$ on the DC bias voltage before and after the neutron irradiation.

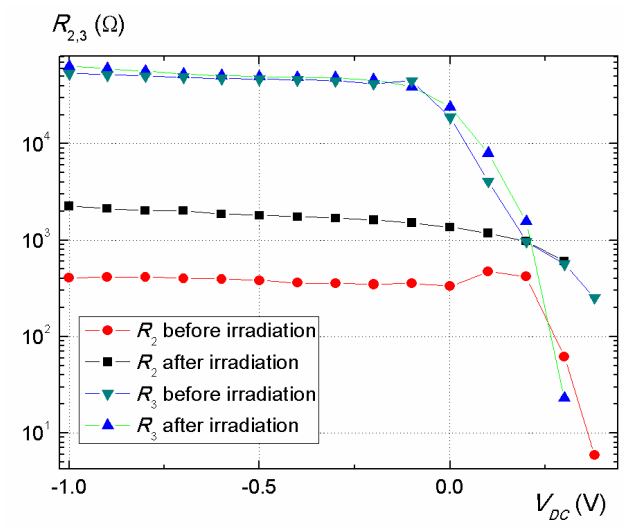

Fig. 6. Parallel resistances as a function of the applied bias before and after neutron irradiation.

In accordance with other studies [18, 19] we suggest that resistance $R_{2}$ is the transport resistance and $R_{3}$ is the recombination resistance. As it is shown in Fig.6, both parallel resistances $R_{2}$ and $R_{3}$ of the AC equivalent circuit are at higher forward bias voltage strongly dependent on the applied DC voltage. They are heading to negligible values and behaviour of the structure is controlled by the series resistance. The effect of the neutron irradiation regarding $R_{3}$ was minimal.

\section{CONCLUSION}

The influence of the irradiation by neutrons on the behaviour of the diode heterostructures $\mathrm{SiC}-\mathrm{Si}$ intended for a photovoltaic application was studied. From the DC analysis it was confirmed that the neutron radiation leads to increase a series resistance and increase of structural defects reflected in the increase in the diode ideality factor. The impedance spectroscopy on the devices a$\mathrm{SiC} / \mathrm{c}-\mathrm{Si}$ over a range of applied biases was performed. An equivalent circuit, consisting of a series resistance, transport resistance, device capacitance, recombination resistance and CPE was proposed and used to identify and quantify the responsible physical mechanisms. The structural defects induced by the neutron particles radiation affect mainly trapping mechanism illustrated by the radiation influence on the CPE included into the equivalent circuit.

\section{ACKNOWLEDGMENT}

This work was supported by the Slovak Research and Development Agency under the contract No. APVV-150326. This work was also supported by the Scientific Grant Agency of the Ministry of Education of the Slovak Republic and of the Slovak Academy of Sciences No. $1 / 0651 / 16$. This paper is also the result of the project implementation: Finalizing of the National Centre for Research and Application of renewable Energy Sources, ITMS: 26240120028, supported by the R\&D Operational Programme funded by the ERDF.

\section{REFERENCES}

[1] M. Zeman and Z. Dong, Heterojunction silicon based solar cells, Physics and technology of amorphous-crystalline heterostructure silicon solar cells, Springer Berlin Heidelberg, 2012.

[2] L. Bofei, B. Lisha, Z. Xiaodan, Z. Dekun, W. Changchun, S. Jian, H. Qian, Xinliang, et. al., "Light management in hydrogenated amorphous silicon germanium solar cells," Solar Energy Materials and Solar Cells, vol. 128, pp. 1-10, September 2014. https://doi.org/10.1016/j.solmat.2014.05.008

[3] F. Janíček, I. Darul'a, J. Gaduš, E. Regula, M. Smitková, L. Polonec, J. Ludvík, J. Kubica, Renewable Energy Sources 1., Pezinok: Renesans, s.r.o., 171 p. ISBN 978-80-89402-05-2, (2009).

[4] K. Masuko, et al., "Achievement of more than $25 \%$ conversion efficiency with crystalline silicon heterojunction solar cell," Photovoltaics, IEEE Journal, vol. 6, 2014. https://doi.org/10.1109/jphotov.2014.2352151

[5] H. Johnston, "Radiation damage of electronic and optoelectronic devices in space," in Proceedings of the 4th International Workshop on Radiation Effects on Semiconductor Devices for Space Application, Tsukuba, Japan, October 2000.

[6] B. Simić, D. Nikolić, K. Stanković, L. Timotijević, and S. Stanković, "Damage Induced by Neutron Radiation on Output Characteristics of Solar Cells, Photodiodes, and Phototransistors," International Journal of Photoenergy, vol. 2013 pp. 1-62013.

[7] M. Perný et al., "Electrical Properties of Solar Heterojunction aSiC/c-Si Irradiated by Neutrons," in Power Engineering 2016. Renewable Energy Sources 2016: 6th International Scientific Conference OZE 2016. Tatranské Matliare, Slovakia, May 31 June 2, 2016. Bratislava : Slovak University of Technology in Bratislava, 2016, ISBN 978-80-89402-81-6

J. R. Srour, C. J. Marshall, and P. W. Marshall, "Review of displacement damage effects in silicon devices," IEEE Transactions on Nuclear Science, vol. 50, no. 3, pp. 653-670, 2003. https://doi.org/10.1109/TNS.2003.813197

[8] C. L. Axness, B. Kerr, and E. R. Keiter, "Analytic 1-D pn junction diode photocurrent solutions following ionizing radiation and including time-dependent changes in the carrier lifetime from a nonconcurrent neutron pulse," IEEE Transactions on Nuclear Science, vol. 57, no. 6, pp. 3314-3321, 2010.

[9] G. Garcia-Belmonte, J. Bisquert, V. Caselles, "The small signal ac impedance of a short p-n junction diode," Sol. St. Electron. 42, p. 939, 1998. https://doi.org/10.1016/S0038-1101(98)00113-0

[10] M. Perný, V.Šály, J. Huran, M. Mikolášek, M. Váry, "Heterojunction a-SiC/c-Si solar cell structures and their electrical characterization," in 31st European photovoltaic solar energy conference and exhibition: Proceedings. Hamburg, Germany. München: WIP, 2015, pp. 683-687, 14 - 18 September 2015.

[11] P. Malkeshkumar, I. Mukhopadhyay, R. Abhijit, "Study of the junction and carrier lifetime properties of a spray-deposited CZTS thin-film solar cell," Semiconductor Science and Technology, vol. 28, 055001 pp.1-7, 2013. 
[12] H. Asil, K. Çinar, E. Gür, C. Coşkun, S. Tüzemen, “Temperature dependent current-voltage characteristics of electrodeposited $\mathrm{p}$ $\mathrm{ZnO} / \mathrm{n}-\mathrm{Si}$ heterojunction," International Journal of Physical Sciences, vol. 8(10), pp. 371-379, 16 March, 2013.

[13] R.N., Chih-Tang Sah, Noyce, W. Shockley, "Carrier Generation and Recombination in P-N Junctions and P-N Junction Characteristics," Proceedings of the IRE, vol. 45, no. 9, pp. 12281243, Sept. 1957. https://doi.org/10.1109/JRPROC.1957.278528

[14] O. Breitenstein, et. al, "Interpretation of the commonly observed I$\mathrm{V}$ characteristics of $\mathrm{c}-\mathrm{Si}$ cells having ideality factor larger than two", in Proc. 21st European Photovoltaics Energy Conf., ed J Poortmans, H Ossenbrink, E Dunlop and P Helm (Munich: Germany) pp. 625-28, 2006.

https://doi.org/10.1109/wcpec.2006.279597
[15] E. Barsoukov and J. R. Macdonald, Impedance Spectroscopy: Theory, Experiment, and Applications. 2nd edition, Impedance Spectroscopy (John Wiley \& Sons, 2005).

[16] C. H., Hsu and F. Mansfeld, "Concerning the conversion of the constant phase element parameter $\mathrm{Y}_{0}$ into a capacitance," Corrosion 57, p. 747, 2001. https://doi.org/10.5006/1.3280607

[17] B. J. Leever, C. A. Bailey, T. J. Marks, M. C. Hersam, M. F. Durstock, "In situ characterization of lifetime and morphology in operating bulk heterojunction organic photovoltaic devices by impedance spectroscopy," Adv.Energy Mater. 2, pp. 120-128, 2012. https://doi.org/10.1002/aenm.201100357

[18] J. Bisquert and G. Garcia-Belmonte, "On Voltage, Photovoltage, and Photocurrent in Bulk Heterojunction Organic Solar Cells," $J$. Phys. Chem. Lett. 2, pp. 1950-1964, 2011. https://doi.org/10.1021/jz2004864 